\title{
On the use of the generalized SPRT method in the equivalent hard sphere approximation for nuclear data evaluation
} \author{
Peter Schillebeeckx ${ }^{3}$, Ivan Sirakov ${ }^{4}$, and Pierre Tamagno ${ }^{1}$ \\ 1 CEA, DEN, Cadarache, 13108 Saint-Paul-les-Durance, France \\ 2 IAEA, Nuclear Data Section, 1400 Vienna, Austria \\ 3 EC-JRC, 2440 Geel, Belgium \\ ${ }^{4}$ INRNE, 1784 Sofia, Bulgaria
}

Gilles Noguere ${ }^{1, a}$, Pascal Archier ${ }^{1}$, Olivier Bouland ${ }^{1}$, Roberto Capote ${ }^{2}$, Cyrille De Saint Jean ${ }^{1}$, Stefan Kopecky ${ }^{3}$,

\begin{abstract}
A consistent description of the neutron cross sections from thermal energy up to the $\mathrm{MeV}$ region is challenging. One of the first steps consists in optimizing the optical model parameters using average resonance parameters, such as the neutron strength functions. They can be derived from a statistical analysis of the resolved resonance parameters, or calculated with the generalized form of the SPRT method by using scattering matrix elements provided by optical model calculations. One of the difficulties is to establish the contributions of the direct and compound nucleus reactions. This problem was solved by using a slightly modified average R-Matrix formula with an equivalent hard sphere radius deduced from the phase shift originating from the potential. The performances of the proposed formalism are illustrated with results obtained for the ${ }^{238} \mathrm{U}+\mathrm{n}$ nuclear systems.
\end{abstract}

\section{Introduction}

Experimentally we can observe that there is a continuous transition from the extreme situation of well-isolated resonances to complete overlap. This transition was first described by Feshbach et al. [1], explaining how the R-Matrix and optical models may coexist between the "resonance region" and the "continuum region".

The main ingredients of the theory of average cross section are given in this document. Equations are then slightly modified to account for direct reactions by introducing complex background terms in the ReichMoore approximation of the R-Matrix theory [2]. Consequences on the neutron strength functions calculated in the hard sphere approximation are discussed with results provided by the OPTMAN code.

\section{Theory of average cross section}

For the sake of clarity, we define $U_{c}, \bar{U}_{c}$ and $\mathcal{S}_{c}$ the elements of the scattering matrix respectively used in the Resolved Resonance Range, in the Unresolved Resonance Range and in the "continuum" for $c=c$ '. Historically, $\bar{U}_{c}$ is called the average collision function by analogy with the collision matrix $U_{c}$ involved in the R-Matrix theory.

In the resonance region, the elements of $U_{c}$ exhibit rapid fluctuations coming from the numerous close-spaced resonances of the compound nucleus. An average value of $U_{c}$ can be defined over an interval $\Delta E$ containing many overlapping levels. This condition is written as:

$$
\Delta E \gg D_{c},
$$

\footnotetext{
a e-mail: gilles.noguere@cea.fr
}

where $D_{c}$ stands for the average spacing of the compound levels. If the interval $\Delta E$ is small enough to assume constant the phase shift $\phi_{c}$, the penetration factor $P_{c}$ and the reduced $\mathrm{R}$-function $R_{c}$, it follows that the analytical averaging of the collision matrix $U$ in terms of average resonance parameters yields a diagonal collision matrix $\bar{U}$ with elements $[3,4]$ :

$$
\bar{U}_{c} \simeq \mathrm{e}^{-2 i \phi_{c}} \frac{\mathcal{B}_{c}+i P_{c} \bar{R}_{c}}{\mathcal{B}_{c}-i P_{c} \bar{R}_{c}} .
$$

The factor $\mathcal{B}_{c}$ depends on boundary condition parameters, which choice is often a matter of convenience [5]. In the present work a "standard" formulation is used, for which $\mathcal{B}_{c}=1$ [6]. Under these conditions, Eq. (2) becomes:

$$
\bar{U}_{c} \simeq \mathrm{e}^{-2 i \phi_{c}} \frac{1+i P_{c} \bar{R}_{c}}{1-i P_{c} \bar{R}_{c}} .
$$

In that case, the average value of the total and shape elastic cross sections can be evaluated as follow:

$$
\begin{gathered}
\bar{\sigma}_{t_{c}} \simeq g_{J} \frac{2 \pi}{k^{2}}\left(1-\operatorname{Re}\left[\bar{U}_{c}\right]\right), \\
\bar{\sigma}_{e_{c}} \simeq g_{J} \frac{\pi}{k^{2}}\left|1-\bar{U}_{c}\right|^{2} .
\end{gathered}
$$

The neutron transmission coefficients are given by:

$$
\mathcal{T}_{c}=1-\left|\overline{U_{c}}\right|^{2} .
$$

\section{Average reduced R-function}

In this work, the average reduced R-function $\bar{R}_{c}$ has been determined from the reduced R-function given by the 
Reich-Moore approximation of the R-Matrix theory [2]:

$$
R_{c}(E)=\sum_{\lambda} \frac{\gamma_{\lambda n_{c}}^{2}}{E_{\lambda}-E-i \Gamma_{\lambda \gamma_{c}} / 2}+\mathcal{R}_{c}(E),
$$

in which $E_{\lambda}$ is the resonance energy, $\gamma_{\lambda n_{c}}$ is the reduced neutron width amplitude and $\Gamma_{\lambda \gamma_{c}}$ is the partial radiation width of the compound state $\lambda$. Lynn derived an expression in which the background term $\mathcal{R}_{c}$ is complex [7]:

$$
\begin{aligned}
R_{c}(E)= & \sum_{\lambda} \frac{\gamma_{\lambda n_{c}}^{2}}{E_{\lambda}-E-i \Gamma_{\lambda \gamma_{c}} / 2}+R_{c}^{\infty}(E) \\
& +R_{c}^{\mathrm{loc}}(E)+i \pi s_{c}^{\mathrm{loc}}(E) .
\end{aligned}
$$

The real part of $\mathcal{R}_{c}$ can be split into the contributions of neighboring $\left(R_{c}^{\text {loc }}\right)$ and far-off levels $\left(R_{c}^{\infty}\right)$. Feshbach [1] assumes that only the immediate-neighbor resonances $R_{c}^{\text {loc }}$ contribute appreciably. He suggests that such a contribution may formally be replaced by a sum over an infinite number of external levels $\mu$, having negative or positive energies. As the consequence, the contribution of these "fictitious" resonances can be included in the sum over levels $\lambda$ of the reduced R-matrix (Eq. (7)):

$$
R_{c}(E) \simeq \sum_{\eta=\lambda, \mu} \frac{\gamma_{\eta n_{c}}^{2}}{E_{\eta}-E-i \Gamma_{\eta \gamma_{c}} / 2}+R_{c}^{\infty}(E)+i \pi s_{c}^{\mathrm{loc}}(E) .
$$

Throughout the R-matrix theory, $R_{c}^{\infty}$ is called the distant level parameter. Its value is lower than unity. Lynn indicates that the far away contribution $R_{c}^{\infty}$ modifies the channel radius $a_{c}$ to give the effective hard-sphere potential scattering radius $R_{c}^{\prime}[8]$. Denoting $R^{\prime}=R_{c}^{\prime}$ for the s-wave channel, one obtains:

$$
R^{\prime}=a_{0}\left(1-R_{0}^{\infty}\right)
$$

The imaginary part $\operatorname{Im}\left[\mathcal{R}_{c}\right]=\pi s_{c}^{\text {loc }}$ modifies the absorption cross section and adds a contribution that is inversely proportional to the velocity.

In the uniform level approximation, where the amplitude $\gamma_{\eta n_{c}}^{2}$ are all equal and the levels $\eta$ are uniformly spaced by an amount $D_{c}$, the average value of the reduced R-function $R_{c}$ is:

$$
\begin{aligned}
\bar{R}_{c} & \simeq \int_{a}^{b} \frac{s_{c}}{E^{\prime}-z} \mathrm{~d} E^{\prime}+\frac{1}{\Delta E} \int_{a}^{b} R_{c}^{\infty}\left(E^{\prime}\right) \mathrm{d} E^{\prime} \\
& +\frac{i \pi}{\Delta E} \int_{a}^{b} s_{c}^{\mathrm{loc}}\left(E^{\prime}\right) \mathrm{d} E^{\prime},
\end{aligned}
$$

with

$$
z=E+i \frac{\Gamma_{\gamma}}{2}
$$

in which $\Gamma_{\gamma}$ represents the average radiation width. The first integral of Eq. (11) is called the Stieltjes transform of the pole strength function $s_{c}$, where $s_{c}$ is a probability density function defined as the ratio of the average reduced width to level spacings:

$$
s_{c}=\frac{\left\langle\gamma_{n_{c}}^{2}\right\rangle}{D_{c}}
$$

The method used by Thomas [3] for assigning a value to the Stieltjes transform of the pole strength function relies on the Sokhotsky's formula. The latter state that:

$$
\lim _{x \rightarrow 0} \frac{1}{x \mp i \varepsilon}=\mathcal{P}\left(\frac{1}{x}\right) \pm i \pi \delta(x),
$$

where $\delta(x)$ is the delta function and $\mathcal{P}$ is the Cauchy principal value of the inverse function $1 / x$. According to the expression (11), we have $\varepsilon=\Gamma_{\gamma} / 2$ and $x=E^{\prime}-E$. The Sokhotsky's formula can be applied if $\Gamma_{\gamma}$ is small. This condition is satisfied in the unresolved resonance range of many isotopes, such as actinides, for which the average radiation widths is lower than $50 \mathrm{meV}$. As a consequence, the identity (14) can be introduced in Eq. (11) to give:

$$
\begin{aligned}
\bar{R}_{c} \simeq & i \pi s_{c}+\underbrace{\mathcal{P} \int_{a}^{b} \frac{s_{c}}{E^{\prime}-E} \mathrm{~d} E^{\prime}+\frac{1}{\Delta E} \int_{a}^{b} R_{c}^{\infty}\left(E^{\prime}\right) \mathrm{d} E^{\prime}}_{\bar{R}_{c}^{\infty}} \\
& +i \pi \underbrace{\frac{1}{\Delta E} \int_{a}^{b} s_{c}^{\text {loc }}\left(E^{\prime}\right) \mathrm{d} E^{\prime}}_{\bar{s}_{c}^{\text {loc }}} .
\end{aligned}
$$

This mathematical trick allows to distinguish, without much complication, the "statistical R-function" $i \pi s_{c}$ and the background contribution $\bar{R}_{c}^{\infty}+i \pi \bar{s}_{c}^{\text {loc }}$ :

$$
\bar{R}_{c} \simeq \bar{R}_{c}^{\infty}+i \pi\left(s_{c}+\bar{s}_{c}^{\mathrm{loc}}\right) .
$$

In the framework of the average R-Matrix approximation, the parameter $\bar{R}_{c}^{\infty}$, the pole strength function $s_{c}$ and the background term $\bar{s}_{c}^{\text {loc }}$ are free parameters. Prior values can be deduced from optical model parameters by using the SPRT method.

\section{Average R-Matrix parameters}

The SPRT method is used to study the consistency between the average R-Matrix parameters adjusted on experimental data and those given by optical model calculations. The standard SPRT method [9] was developed for s- and p-wave parameters $(l=0,1)$. The method was generalized in order to provide pole strength functions and parameter $\bar{R}_{c}^{\infty}$ for higher order partial wave $(l \geq 1)$ [10]. In practice, the analytically averaged collision matrix (3) can be equated to the optical model S-Matrix $\mathcal{S}_{c}$ expressed for further convenience through the diagonal $\mathrm{C}$-matrix:

$$
\mathcal{S}_{c}=1+2 i C_{c} \text {. }
$$

Thus, from Eqs. (3) and (17) one obtains the pole strength functions and distant level parameter $\bar{R}_{c}^{\infty}$ :

$$
P_{c} \bar{R}_{c}^{\infty}=\frac{2 \alpha_{c} \cos \left[2 \phi_{c}\right]+\left(1-2 \beta_{c}\right) \sin \left[2 \phi_{c}\right]}{1+2 \theta_{c}^{2}-2 \beta_{c}+\left(1-2 \beta_{c}\right) \cos \left[2 \phi_{c}\right]-2 \alpha_{c} \sin \left[2 \phi_{c}\right]},
$$

$$
\begin{aligned}
& \pi P_{c}\left(s_{c}+\bar{s}_{c}^{\mathrm{loc}}\right)= \\
& \frac{2\left(\beta_{c}-\theta_{c}^{2}\right)}{1+2 \theta_{c}^{2}-2 \beta_{c}+\left(1-2 \beta_{c}\right) \cos \left[2 \phi_{c}\right]-2 \alpha_{c} \sin \left[2 \phi_{c}\right]} .
\end{aligned}
$$

in which $\alpha_{c}, \beta_{c}$ and $\theta_{c}$ are free parameters. In the ground state channel, for target nucleus with $I=0$, they represent 
the real part, the imaginary part and the absolute value of $C_{c}$ :

$$
\left\{\begin{array}{l}
\alpha_{c}=\operatorname{Re}\left[C_{c}\right] \\
\beta_{c}=\operatorname{Im}\left[C_{c}\right] \\
\theta_{c}=\left|C_{c}\right|
\end{array}\right.
$$

An explicit separation of the pole strength function $s_{c}$ and of the background term $\bar{s}_{c}^{\text {loc }}$ can be achieved by using the neutron transmission coefficients. By introducing Eq. (3) in Eq. (6), the neutron transmission coefficients can be written as follow:

$$
\mathcal{T}_{c}=\frac{4 \pi P_{c}\left(s_{c}+\bar{s}_{c}^{\mathrm{loc}}\right)}{\left[1+\pi P_{c}\left(s_{c}+\bar{s}_{c}^{\mathrm{loc}}\right)\right]^{2}+P_{c}^{2} \bar{R}_{c}^{\infty^{2}}} .
$$

The latter expression can be split in two contributions:

$$
\begin{aligned}
\mathcal{T}_{c}= & \underbrace{\frac{4 \pi P_{c} s_{c}}{\left[1+\pi P_{c}\left(s_{c}+\bar{s}_{c}^{\mathrm{loc}}\right)\right]^{2}+P_{c}^{2} \bar{R}_{c}^{\infty^{2}}}}_{T_{c}} \\
& +\underbrace{\frac{4 \pi P_{c} \bar{s}_{c}^{\mathrm{loc}}}{\left[1+\pi P_{c}\left(s_{c}+\bar{s}_{c}^{\mathrm{loc}}\right)\right]^{2}+P_{c}^{2} \bar{R}_{c}^{\infty^{2}}}}_{T_{D_{c}}} .
\end{aligned}
$$

For spherical or weakly deformed nuclei, the contribution of the direct reactions $T_{D_{c}}$ disappears $\left(\bar{s}_{c}^{\text {loc }}=0\right)$, and the expression of the neutron transmission coefficient becomes:

$$
\mathcal{T}_{c}=T_{c}=\frac{4 \pi P_{c} s_{c}}{\left(1+\pi P_{c} s_{c}\right)^{2}+P_{c}^{2} \bar{R}_{c}^{\infty^{2}}}
$$

By using the notation

$$
f=\frac{1}{\left(1+\pi P_{c} s_{c}\right)^{2}+P_{c}^{2} \bar{R}_{c}^{\infty^{2}},}
$$

the expression (23) can be rewritten as:

$$
T_{c}=4 \pi P_{c} s_{c} f
$$

The latter result indicates that the neutron transmission coefficient and the penetration factor differ by a factor equal to $4 \pi s_{c} f$. In Ref. [11], $f$ was also introduced to account for the difference in reflexion between a diffuse-edge optical potential and its equivalent squarewell. The latter study mainly suggests to replace the channel radius $a_{c}$ involved in the R-Matrix formalism by an equivalent hard-sphere radius deduced from the phase shift originating from the potential.

\section{Hard-sphere approximation}

The channel radius is one of the boundary conditions introduced in the R-matrix and Optical Model reaction formalisms to match the solution of the Schrodinger equation with its corresponding expression valid outside the region of nuclear forces. Such an abrupt separation of the configuration space by an imaginary closed surface of radius $a_{c}$ leads to the notions of "internal" and "external" regions [12]. The complex mean-field potential $V(r)$ vanishes in the external region. The size of the internal

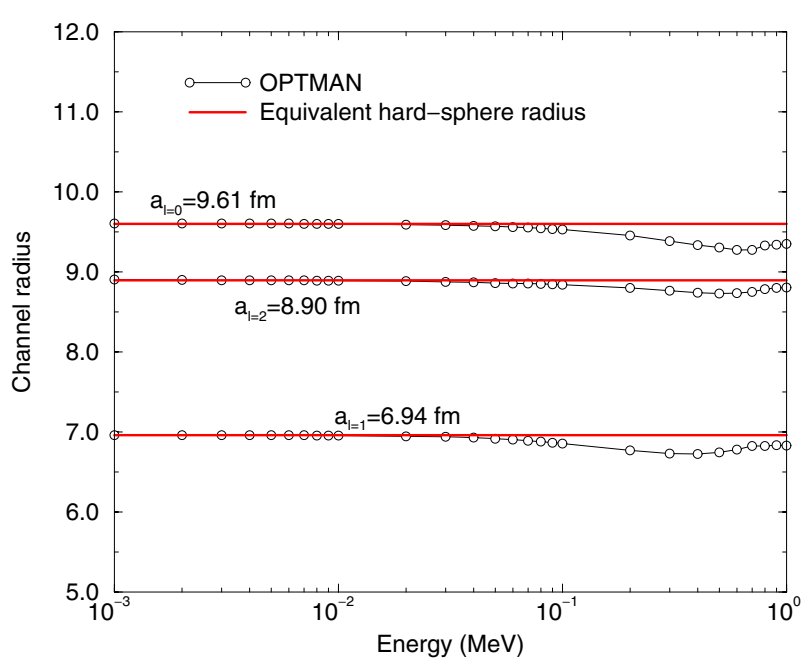

Figure 1. Channel radii for ${ }^{238} \mathrm{U}+\mathrm{n}$ calculated with the OPTMAN code by using Eq. (26) with optical model parameters of Ref. [13].

region is not defined. Therefore, the channel radii are more or less chosen arbitrarily. In this work, this ambiguity is solved by choosing $a_{c}$ such that the optical model and its equivalent square-well provide the same phase shifts at the common channel radii. The resonance theory determines the hard-sphere phase shifts $\phi_{l}$ from the precisely known radial wave functions at the channel radius $a_{c}$. Denoting $\rho=k a_{c}$, one obtains:

$$
\left\{\begin{array}{l}
\phi_{0}(\rho)=\rho, \\
\phi_{1}(\rho)=\rho-\tan ^{-1}(\rho), \\
\phi_{2}(\rho)=\rho-\tan ^{-1}\left(\frac{3 \rho}{3-\rho^{2}}\right),
\end{array}\right.
$$

Figure 1 shows the channel radii calculated with Eq. (26) by using the phase shifts provided by the OPTMAN code with a set of optical model parameters recently established for the nuclear system ${ }^{238} U+n$ [13]. A good agreement is observed with the equivalent hardsphere radii up to $150 \mathrm{keV}$, which is a satifactory upper energy limit for the analysis of the Unresolved Resonance Range of the neutron cross sections.

Equations (18), (19) and (22) were implemented in the OPTMAN code for calculating the neutron strength functions and distant level parameters for $l=0,1,2$. Results obtained for ${ }^{238} \mathrm{U}+\mathrm{n}$ are reported in Fig. 2. They were obtained by using the same set of optical model parameters as used in Fig. 1 together with the equivalent hard-sphere radii $a_{0}=9.61 \mathrm{fm}, a_{1}=6.94 \mathrm{fm}$ and $a_{2}=$ $8.90 \mathrm{fm}$. Similar calculations were repeated by replacing the $l$-dependent equivalent hard-sphere radii by a single channel radius calculated with the ENDF convention [14]:

$$
a_{c}=1.23 A^{1 / 3}+0.8(\text { in fm }) \text {. }
$$

For the nuclear system ${ }^{238} \mathrm{U}+\mathrm{n}$, the channel radius in the ENDF convention is $a_{c}=8.42 \mathrm{fm}$. In Fig. 2, we can observe that the distant levels parameters vanish $\left(\bar{R}_{c}^{\infty} \simeq 0\right)$ when the equivalent hard sphere radii are used. Consequently, Eq. (10) indicates that the channel radius $a_{c}$ becomes strictly equivalent to the potential scattering radius $R^{\prime}$. As stated by Vogt in the 90s, the equivalent hardsphere radius becomes the "natural" choice of $a_{c}$ for each 

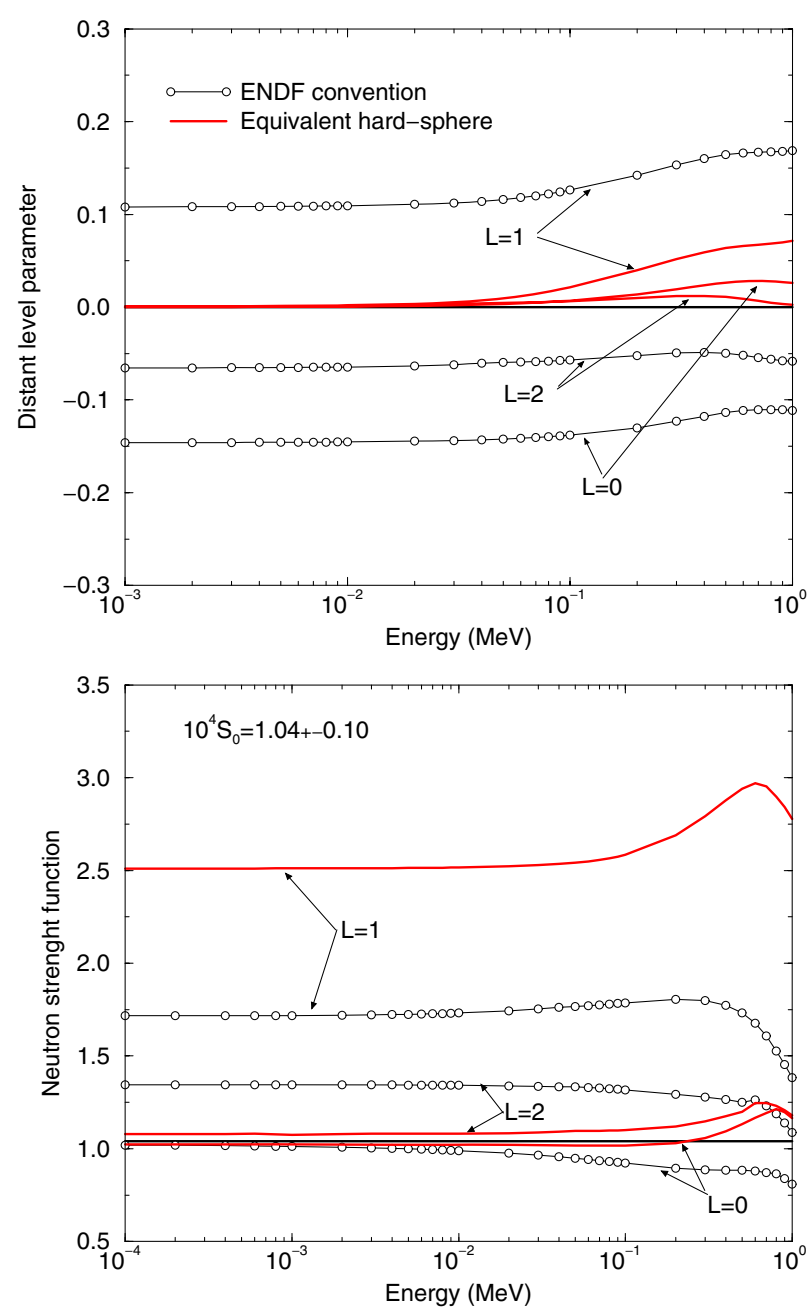

Figure 2. Distant level parameters and neutron strength functions for ${ }^{238} \mathrm{U}+\mathrm{n}$ calculated with the OPTMAN code by using Eqs. (18), (19) and (22) with optical model parameters of Ref. [13]. The solid-dot lines were obtained with the channel radius calculated with Eq. (27), and the solid lines with the channel radii of Fig. 1.

reaction channel [15]. For s-wave channels, it represents the "effective" radius $R^{\prime}$ of the target at zero energy:

$$
R^{\prime}=a_{l=0}
$$

The main conclusion of this work is that the SPRT method yields s-wave and d-wave neutron strength functions of similar magnitude and nearly independent of the neutron energy:

$$
S_{0} \simeq S_{2} \simeq \mathrm{cst},
$$

if and only if the optical model and its equivalent squarewell provide the same phase shifts at the common channel radii $a_{c}$. These results provide a mathematical framework for the "rule of thumb" often used by Fröhner and Bouland [16], which defines the behavior of the neutron strength functions for odd and even angular momentum l. For higher-order partial waves, this empirical rule says that the strength functions for $l=0,2,4, \ldots$ are similar, and those for $l=1,3,5, \ldots$ are likewise similar.

\section{Conclusions}

Average R-Matrix parameters (neutron strength function and distant level parameter) were determined by focusing our analysis on the conspicuous role of the channel radius $a_{c}$. This parameter is one of the boundary condition introduced in the R-matrix theory assuming an abrupt division of the configuration space. The resonance theory has some undesirable features of the square-well potential for which $a_{c}$ is chosen more or less arbitrarily. The use of equivalent hard sphere radii, deduced from phase shifts provided by optical model calculations, shows that the contribution of the distant level parameters vanishes in the average R-Matrix formalism. As a consequence, the effective radius $R^{\prime}$ and the channel radius $a_{0}$ for resonances having zero neutron orbital angular momentum $(l=0)$ are the same quantity. It must be emphasized that, for ${ }^{238} \mathrm{U}+\mathrm{n}$, this property leads to s- and d-wave neutron strength functions of similar magnitude $\left(S_{0} \simeq S_{2}\right)$. The OPTMAN results confirm that such lowenergy neutron spectroscopic information could be a strong constraint in the optimization procedure of the optical model parameters.

\section{References}

[1] H. Feshbach, C.E. Porter and V.F. Weisskopf, Phys. Rev. 96, 448 (1954)

[2] A.M. Lane and R.G. Thomas, Rev. Mod. Phys. 30, 257 (1958)

[3] R.G. Thomas, Phys. Rev. 97, 224 (1955)

[4] P.A. Moldauer, Phys. Rev. 129, 754 (1963)

[5] F.C. Barker, Aust. J. Phys. 25, 341 (1972)

[6] I. Sirakov, B. Becker, R. Capote, E. Dupont, S. Kopecky, C. Massimi and P. Schillebeeckx, Eur. Phys. J. A 49, 144 (2013)

[7] J.E. Lynn, The Theory of Neutron Resonance Reactions (Clarendon Press, Oxford, 1968)

[8] J.E. Lynn, Proc. Phys. Soc. 82, 903 (1963)

[9] J.-P. Delaroche, Ch. Lagrange and J. Salvy, in: Proceedings of a Consultants Meeting on the Use of Nuclear Theory in Neutron Nuclear Data Evaluation, Trieste, Italy, 1975; See also IAEA-190, International Atomic Energy Agency Nuclear Data Services (1976)

[10] E. Rich et al., Nucl. Sci. Eng. 162, 76 (2009)

[11] G. Michaud, L. Scherk and E. Vogt, Phys. Rev. C 1, 864 (1970)

[12] P.L. Kapur and R. Peierls, Proc. Roy. Soc. (Lond.) A 166, 277 (1938)

[13] E.Sh. Soukhovitskii, R. Capote, J.M. Quesada, S. Chiba, and D.S. Martyanov, Phys. Rev. C 94, 064605 (2016)

[14] M. Herman, ENDF-102 Data Formats and Procedures for the Evaluated Nuclear Data File ENDF-6, Brookhaven National Laboratory Report BNL-NCS44945-05-Rev, 2005

[15] E. Vogt, Phys. Lett. B 389, 637 (1996)

[16] F.H. Frohner and O. Bouland, Nucl. Sci. Eng. 137, 70 (2001) 